\section{Line slope vs line arrangement: A reply to Pomerantz and Sager}

\author{
JACOB BECK \\ University of Oregon \\ Eugene, Oregon 97403
}

Similarity grouping (i.e., the segmentation of a field into units on the basis of differences between textural elements) occurs strongly in terms of simple properties such as brightness, size, and the slopes of lines composing figures (Beck, 1967, 1972; Olson \& Attneave, 1970). More complex properties such as differences in the arrangement of the lines of textural elements will not in general produce strong similarity grouping. I have also hypothesized that the effectiveness of stimulus differences in producing similarity grouping corresponds to their extrafoveal discriminability in a patterned visual field when attention is distributed over the field and is not focused (Beck, 1972). In one series of experiments (Beck, 1972, 1974), I placed four figures at each of the corners of an imaginary square. Either all four were the same or one of the figures was different. The subject's task was to detect whether a disparate figure was present. Subjects made fewer errors when figures differed in the slopes of the lines composing the figures (e.g., a $T$ and a tilted $T$ ) than when they differed in the arrangement of the lines (e.g., a $\mathrm{T}$ and an $\mathrm{L}$ ). When a single figure was presented in an empty visual field and attention could be focused, the discriminability of a difference in line arrangement and of a difference in line slope was similar. Ambler and I confirmed that the discrimination of a difference in line slope is better than that of a difference in line arrangement in the periphery when attention is distributed but that with focused attention their discriminability becomes similar (Beck \& Ambler, 1972, 1973). Ambler and Finklea (1976) have shown that with foveal vision a difference in line slope may also be picked up more readily than a difference in line arrangement under distributed attention if the visual system is taxed by increasing the number of figures in the field.

Pomerantz and Sager (1976) have questioned whether the detection of stimulus differences with distributed attention is better for differences in line slope than for differences in line arrangement. They reported an experiment using the procedure similar to Beck's in which four elements were placed in the corners of an imaginary square. They found that the mean reaction time $(\mathrm{RT})$ for discriminating between two diagonal lines ( $\backslash$ vs / ) was $1,884 \mathrm{msec}$. Next, they added lines to each of these diagonals, changing the stimuli into a triangle and an arrow ( $\Delta \mathrm{vs} \bar{N}) .^{1}$ These figures differed both in line slope and in line arrangement. They found that the mean RT for these figures was $749 \mathrm{msec}$. Next, lines were added so that the triangle and arrow had the same line slopes but differed in line arrangement $(\Delta v s K)$. The mean RT for these figures was $759 \mathrm{msec}$. Thus, subjects were able to discriminate between the triangle and arrow more quickly than between the diagonal lines independent of line slope and arrangement. They, therefore, question whether the visual system can be characterized to be more sensitive to differences in line slope than to differences in line arrangement under distributed attention.

First, it should be pointed out that not all differences in line slope are equally effective in producing similarity grouping or are more discriminable than differences in line arrangement. Though an $X$ has the same slopes of lines as a tilted T, Xs both group less readily (Beck, 1967) and are less discriminable (Beck, 1974) than tilted Ts in a pattern of upright Ts. This appears to be because the central symmetry of an $X$ readily causes it to lose discriminability outside the fovea when it subtends a small visual angle (Beck, Note 1). Second, differences in line arrangement under some circumstances will facilitate both grouping and peripheral discriminability. Olson and Attneave (1970), for example, found that sideways $V s$ in a pattern of upright Vs group better than inverted Vs. A sideways $V$ is also more discriminable in a pattern of upright Vs than an inverted $\mathrm{V}$ (Beck, 1972). The reason here appears to be that sideways and upright Vs differentially stimulate vertical and horizontal elliptical receptive fields while upright and inverted Vs do not (Beck, 1972). Sideways Vs $(>)$ have an overall vertical orientation, whereas both upright $(V)$ and inverted $(\Lambda)$ Vs have an overall horizontal orientation.

In connection with the report of Pomerantz and Sager, it needs to be pointed out that, first, a pattern made up of 45- and 135-deg lines produces only moderate grouping and peripheral discriminability. Olson and Attneave (1970) reported that a pattern made up of vertical and horizontal lines gives better grouping than a pattern made up of 45- and 135-deg diagonal lines, even though the differences between the slopes of the lines is $90 \mathrm{deg}$ in both instances. Subjects were also able to discriminate a horizontal line in a pattern of vertical lines a great deal better than a 135-deg line in a pattern of 45-deg lines (Beck, 1972). One possible explanation for the inferiority of 45- and 135-deg lines to produce strong similarity grouping and good peripheral discriminability is that in the periphery vertically and horizontally oriented receptive fields, because of their increased size, are stimulated by both 45 - and 135-deg diagonal lines.

Second, brightness is confounded with arrangement and slope differences in the arrow and triangle 
figures. Pomerantz and Sager, when they added lines to the diagonal lines to make a triangle and arrow also changed the distribution of brightnesses in the figures. A difference in the distribution of slopes has been shown to facilitate both similarity grouping and peripheral discriminability under distributed attention (Beck, 1972). Similarly, differences in the distribution of brightnesses should facilitate both similarity grouping and peripheral discriminability. One can explain the Pomerantz and Sager results by simply assuming that the visual system is sensitive to the dark area at the juncture of the three lines in the arrow stimuli which is not present in the triangle stimuli. What is suggested is that the improved discrimination is not based on the arrangement of the lines but on the dark corner present in the arrow stimuli but not in the triangle stimuli. The ineffectiveness of the slope differences between the diagonal lines, relative to the brightness differences, is consistent with the fact that the discrimination between 45- and 135-deg diagonal lines, as pointed out above, is relatively weak. Figural properties are coupled, and changes will often simultaneously change a number of variables. One must therefore be careful not to confound variables.

\section{REFERENCE NOTE}

1. Beck, J. Similarity grouping and peripheral discriminability under uncertainty II. (Technical Report 233) College Park: University of Maryland Computer Science Center, 1973.

\section{REFERENCES}

Ambler, B. A., \& FinkleA, D. L. The influence of selective attention in peripheral and foveal vision. Perception \& Psychophysics, 1976, 19, 518-524.

BECK, J. Perceptual grouping produced by line figures. Perception \& Psychophysics, 1967, 2, 491-495.

BECK, J. Similarity grouping and peripheral discriminability under uncertainty. American Journal of Psychology, 1972, 85, 1-19.

BECK, J. Relation between similarity grouping and peripheral discriminability. Joumal of Experimental Psychology, 1974, 102. 1145-1147.

BECK, J., \& AMBLER, B. Discriminability of differences in line slope and in line arrangement as a function of mask delay. Perception \& Psychophysics, 1972, 12, 33-38.

BECK, J., \& AMBLER, B. The effects of concentrated and distributed attention on peripheral acuity. Perception \& Psychophysics, 1973, 14, 225-230.

Olson, R. K., \& Attneave, F. What variables produce similarity grouping? American Journal of Psychology, 1970, 83, $1-21$.

Pomerantz, J. R., \& SAger, L. C. Line-slope vs. line-arrangement discrimination: A comment on Ambler and Finklea's paper. Perception \& Psychophysics, 1976, 20, 220.

\section{NOTE}

1. The figures shown by Pomerantz and Sager are $\Delta v s \searrow$. These do not differ in both line slope and line arrangement as described in their article. $I$ have therefore changed the arrow figure to be consistent with their description.

(Received for publication November 12, 1976.) 